\title{
AVALIAÇÃO DA RECONSTRUÇÃO DOTRATO ALIMENTAR COM TUBO GÁSTRICO OU COLÔNICO NA ESOFAGECTOMIA POR CÂNCER DE ESÔFAGO
}

\section{EVALUATION OF THE RECONSTRUCTION TECHNIQUES OF THE ALIMENTARY TRACT WITH GASTRIC OR COLONIC TUBE IN ESOPHAGECTOMY FOR ESOPHAGEAL CANCER}

\author{
Carlos Eduardo Pinto, TCBC-RJ'; Daniel de Souza Fernandes, ASCBC-RJ3; \\ Eduardo Amaral Moura Sá,ACBC-RJ²; Washington Oliveira Telles II ${ }^{2}$; Jurandir Almeida Dias, TCBC-RJ ${ }^{4}$
}

\begin{abstract}
RESUMO: Objetivo: Relatar a experiência com as principais técnicas de reconstrução do trato alimentar após esofagectomia por câncer de esôfago. Metodos: Foram analisados retrospectivamente 68 pacientes submetidos à esofagectomia entre fevereiro de 1997 e novembro de 2005. Todos os pacientes incluídos no estudo foram submetidos à esofagectomia com reconstrução com tubo gástrico ou colônico e anastomose cervical. Resultados: A idade média foi de 55,4 anos (25-74 anos), 50 pacientes eram do sexo masculino e 18 pacientes do sexo feminino, 27 pacientes apresentavam o tumor localizado no esôfago médio e 41 pacientes no esôfago distal, sendo carcinoma epidermóide em 35 pacientes e adenocarcinoma em 33 pacientes. A ressecção foi por via transtorácica em 35 indivíduos e por via transhiatal em 33. A reconstrução com tubo gástrico se deu em 58 pacientes e com tubo colônico em 10 pacientes. A morbidade total da série foi de 52,9\%. A mortalidade operatória foi de 5,8\%. A sobrevida média foi de 35 meses. Conclusão: A esofagectomia com reconstrução com tubo gástrico e anastomose cervical é factível tecnicamente, sendo um procedimento realizado de rotina nos pacientes portadores de câncer de esôfago com indicação cirúrgica. Utilizamos, e recomendamos, a reconstrução com tubo colônico principalmente nos pacientes com cirurgia prévia no estômago ou quando da necessidade de ressecção ampliada deste, impossibilitando a confecção da reconstrução do trânsito alimentar com a gastroplastia (Rev. Col. Bras. Cir. 2008; 35(6): 368-373).
\end{abstract}

Descritores: Neoplasias esofágicas Trato gastrointestinal; Esofagectomia; Gastroplastia.

\section{INTRODUÇÃO}

O câncer de esôfago corresponde a uma neoplasia maligna de grande relevância, já que se trata de uma enfermidade pouco sintomática e com diagnóstico estabelecido em fases avançadas na maioria dos casos. Isso acarreta elevada mortalidade e prognóstico pouco favorável para os pacientes $^{1,2,3}$.

Como ocorre com outros tipos de tumores, o tratamento a ser empregado no câncer do esôfago, e seus resultados, dependem basicamente do estadiamento. Embora seja potencialmente curável em estágios iniciais, os estágios mais avançados comprometem qualquer opção de cura para estes pacientes, restando-lhes apenas o tratamento paliativo ${ }^{4}$.

$\mathrm{Na}$ ausência de contra-indicação do paciente ao procedimento cirúrgico, a ressecção em bloco envolvendo o esôfago e linfonodos regionais, continua sendo o tratamento padrão para o câncer de esôfago. Os procedimentos cirúrgicos envolvendo este órgão são atualmente realizados com maior segurança, apresentando redução significativa na morbidade e mortalidade operatória ${ }^{5}$.

Ainda existe grande controvérsia na literatura especializada em cirurgias no esôfago, no que diz respeito à via de acesso cirúrgico mais adequada, e a extensão do procedimento cirúrgico, principalmente a radicalidade da linfadenectomia.

A via de acesso ao esôfago interfere na morbimortalidade operatória e na radicalidade cirúrgica. A ressecção transhiatal apresenta como benefício uma maior rapidez na realização da esofagectomia e menor trauma cirúrgico por ser menos invasiva. A via transtorácica apresenta como benefício uma maior facilidade para dissecção do esôfago, com menor risco de lesão iatrogênica e maior radicalidade cirúrgica.

Devido à rica drenagem linfática do esôfago, a maioria dos pacientes apresenta doença avançada com comprometimento linfonodal no momento do diagnóstico ${ }^{6,7}$. Diversos estudos demonstraram que a ressecção em bloco do esôfago e linfonodos regionais é responsável por melhor controle local, com redução da taxa de recidiva e maior sobrevida nos pacientes portadores desta neoplasia com indicação cirúrgica $^{8-12 .}$

Restabelecer a continuidade do trato alimentar após a ressecção do tumor, permitindo uma dieta normal e conseqüente melhor qualidade de vida, é considerado ponto fundamental nas cirurgias para o câncer do esôfago. Essa reconstrução é feita mais comumente com o estômago, poden-

\footnotetext{
1. Cirurgião Oncológico; Doutor e Mestre em Cirurgia pela UFRJ; Coordenador do Grupo de Esôfago do INCA

2. Cirurgião Oncológico.

3. Residente de Cirurgia Oncológica do INCA

4. Chefe da Seção de Cirurgia Abdômino-pélvica do INCA.

Recebido em 04/07/2008

Aceito para publicação em 10/09/2008

Conflito de interesses: nenhum

Fonte de financiamento: nenhuma

Trabalho realizado na Seção de Cirurgia Abdômino-Pélvica, Instituto Nacional de Câncer (INCA-MS).
} 
do também ser utilizado o cólon ou segmento de intestino delgado.

Diante destes fatores o principal objetivo deste estudo é relatar a experiência do Instituto Nacional do Câncer (INCA) na reconstrução do trato alimentar após esofagectomia por câncer de esôfago, em pacientes matriculados na Seção de Cirurgia Abdômino-pélvica do Instituto Nacional de Câncer.

\section{MÉTODO}

Foram analisados retrospectivamente 68 pacientes matriculados no Instituto Nacional do Câncer (INCA) entre fevereiro de 1997 e novembro de 2005. Todos os pacientes incluídos no estudo realizaram esofagectomia subtotal por via transtorácica ou transhiatal e tiveram a reconstrução do trato alimentar com tubo gástrico ou colônico com anastomose cervical. Foram excluídos do estudo os pacientes que realizaram esofagectomia com reconstrução do trato alimentar com o intestino delgado e os pacientes com anastomose intratorácica.

A avaliação do estudo foi feita com base nos prontuários e no Programa de Gestão pela Qualidade Total da Seção de Cirurgia Abdômino-Pélvica. Este programa armazena os dados de todos os pacientes submetidos à intervenção cirúrgica pela Seção de Cirurgia Abdômino-Pélvica.

$\mathrm{O}$ estadiamento oncológico foi realizado segundo normas do TNM (AJCC/UICC) e a avaliação pré-operatória se deu com tomografia computadorizada helicoidal de tórax, abdome e pelve, avaliação da função pulmonar (gasometria arterial e prova de função respiratória) e da função cardíaca (eletrocardiograma e ecocardiograma). Outros exames mais específicos eram realizados de acordo com as necessidades individuais dos pacientes.

A seleção para o tratamento cirúrgico levou em conta o "performance status" (I ou II pela escala de Zubrod - ECOG), presença de doença ressecável na avaliação préoperatória, ausência de doença metastática e condições clinicas adequadas para realização do procedimento. Os pacientes sem os critérios acima foram encaminhados para tratamento quimiorradioterápico exclusivo ou tratamento paliativo.

O acompanhamento pós-operatório foi realizado por equipe multiprofissional, com avaliação médica, fisioterápica, psicológica e nutricional periódica. A avaliação médica pósoperatória se deu a cada três meses no primeiro ano, semestralmente do segundo ao quarto ano e anualmente após o quinto ano.

Os principais parâmetros avaliados no estudo foram: via de acesso cirúrgico (Figuras 1 e 2), tipo de reconstrução do trato alimentar (Figuras 3 e 4), morbidade operatória, mortalidade operatória e sobrevida.

Outros fatores analisados foram à idade, sexo, localização do tumor, tipo histológico, número de linfonodos ressecados, número de linfonodos metastáticos invasão angiolinfática, invasão perineural e o tempo cirúrgico.

Foi definido como morbidade operatória qualquer complicação clínica ou cirúrgica relacionada com a esofagectomia, e mortalidade operatória o óbito ocorrido du-

rante ou até 30 dias após o procedimento cirúrgico. A sobrevida foi calculada pelo método de Kaplan-Meier.

\section{RESULTADOS}

Do total de 68 pacientes, 50 eram do sexo masculino e 18 do sexo feminino, com idade média de 55,4 anos (25-74 anos). Os dados relativos à topografia, ao tipo histológico, à invasão angiolinfática e à invasão neural estão escritos na tabela 1. A média do maior diâmetro tumoral foi de 4,9 cm (1,3$15 \mathrm{~cm})$.

As vias cirúrgicas utilizadas, os tipos de reconstrução, o número de linfonodos dissecados, que foram positivos para doença metastática em 36 pacientes (média de 3,9 linfonodos metastático na série), a média do tempo cirúrgico, a morbidade operatória e a mortalidade operatória estão citados na tabela 2 .

Dentre as complicações técnicas, destacam-se: nove casos de fístula anastomótica cervical, três casos de necrose do tubo gástrico, um caso de brida precoce com necessidade de re-operação e dois casos de hemorragia (um proveniente da artéria brônquica direita e outro da veia jugular interna esquerda).

Dentre as complicações clinicas destacam-se: $15 \mathrm{ca}-$ sos de pneumonia, um caso de empiema pleural, um caso de trombose venosa profunda, dois casos de infecção da ferida operatória e um caso de infecção do trato urinário.

Com relação à sobrevida, observamos uma sobrevida média de 35 meses, sobrevida global em dois anos de $65 \%$ e sobrevida global de cinco anos em 24\%. Quarenta pacientes foram a óbito e 28 pacientes permaneceram vivos até o fechamento do estudo, sendo 22 sem evidência de doença locorregional.

Tabela 1 - Localização, tipo histológico e infiltração dos tumores.

Terço médio

27 casos

Terço inferior

41 casos

Adenocarcinoma

33 casos

Carcinoma epidermóide

35 casos

Invasão angiolinfática

43 casos

Invasão neural

41 casos

Tabela 2 - Dados do ato operatório.

Acesso transtorácico

Acesso transhiatal

Reconstrução com tubo gástrico

Reconstrução com tubo colônico

Média de linfonodos ressecados

Média tempo cirúrgico

Morbidade operatória

Mortalidade operatória
35 casos

33 casos

58 casos

10 casos

$18,9 \operatorname{linf}(0-51)$

$323 \min (180-580)$

$52,9 \%$

$5,8 \%$ 


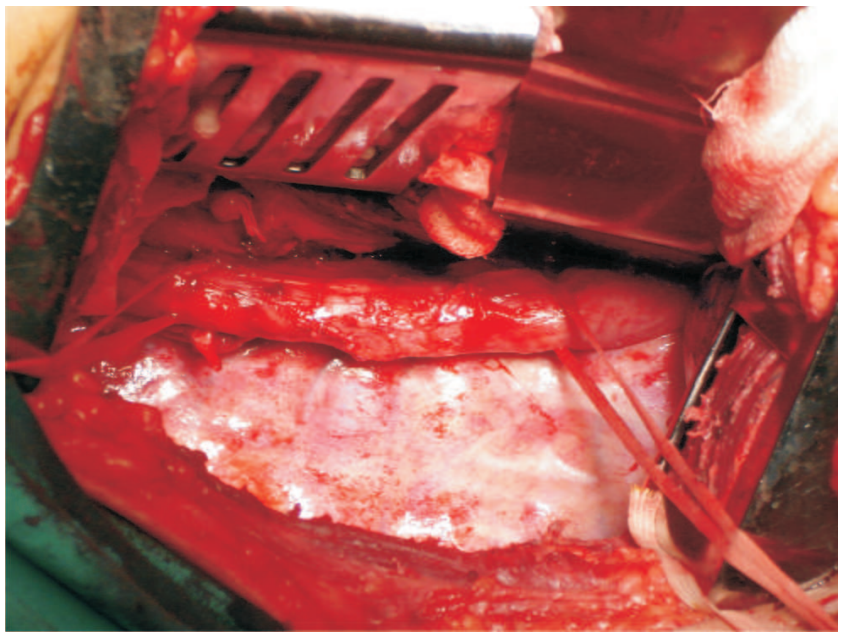

Figura 1 - Via transtorácica.

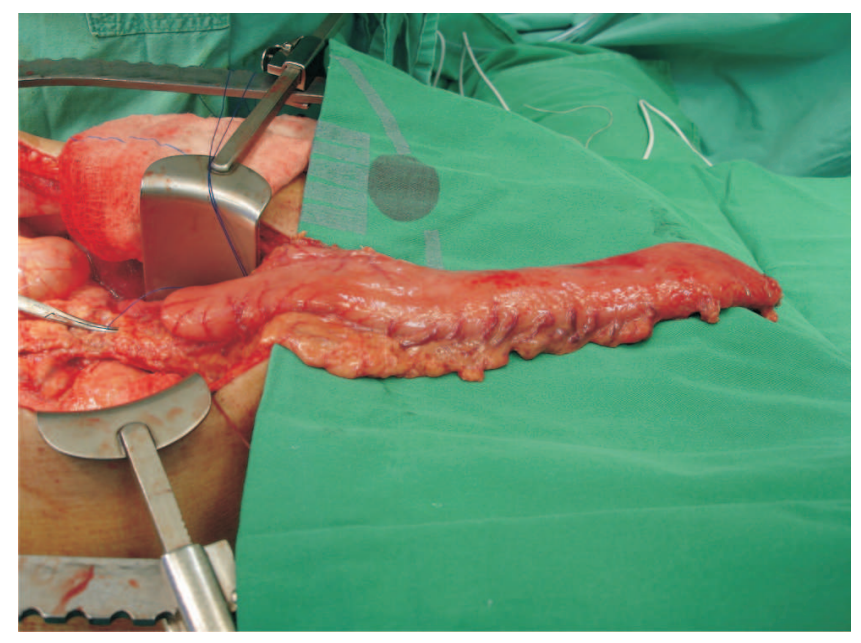

Figura 3 - Tubo gástrico.

\section{DISCUSSÃO}

Têm-se observado nos últimos anos, um aumento considerável na incidência do adenocarcinoma do esôfago distal, principalmente no Ocidente, onde ele já superou o carcinoma epidermóide e se tornou a neoplasia esofagiana mais comum ${ }^{13 .}$ No nosso estudo também observamos esta tendência, tanto que dos 68 pacientes estudados 35 apresentavam carcinoma epidermóide e os outros 33 pacientes adenocarcinoma. Na Ásia, o carcinoma epidermóide do terço médio continua sendo o tumor que mais comumente acomete o esôfago ${ }^{14}$.

A esofagectomia corresponde o tratamento padrão para o câncer de esôfago, tendo índices globais de cura que chegam até a $40 \%$ e mortalidade operatória menor que 5\% nos centros de referência ${ }^{15,16}$. No nosso estudo obtivemos mortalidade operatória de $5,8 \%$, sendo compatível com os outros centros de referência.

A baixa eficácia do tratamento cirúrgico no câncer do esôfago, em termos curativos, é explicada principalmente pela disseminação linfática precoce destes tumores, devido à rica drenagem linfática que se inicia na camada submucosa. Assim

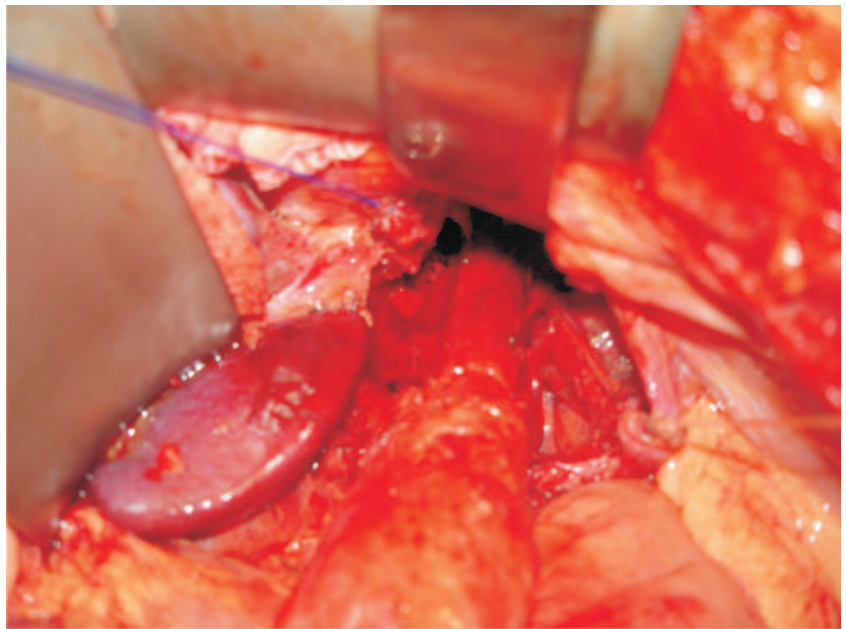

Figura 2 - Via transhiatal.

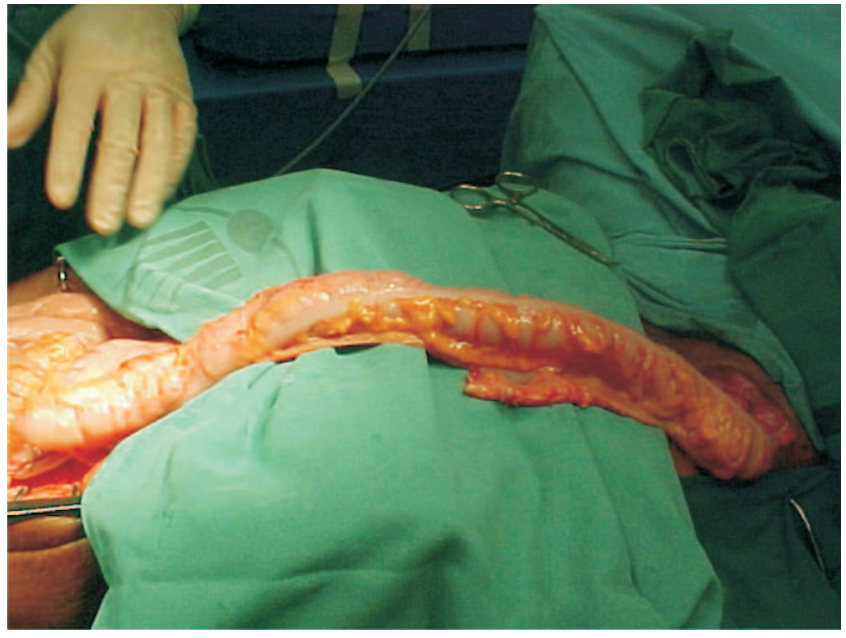

Figura 4 - Tubo colônico.

tumores com invasão de camada submucosa (T1sm) apresentam metástase linfonodal em até $45 \%$ dos $\operatorname{casos}^{17,18}$.

A sobrevida em cinco anos para pacientes com câncer de esôfago é de aproximadamente $50 \%$ no estágio I, $31 \%$ no estádio II, $20 \%$ no estádio III e $4 \%$ no estagio IV. Somente um baixo percentual destes pacientes apresenta, no momento do diagnóstico, doença restrita à mucosa, onde a ressecção cirúrgica possibilita a sobrevida em cinco anos em até $90 \%{ }^{14}$.

Os principais fatores, que utilizamos no nosso grupo de esôfago, e que devem ser considerados quando se programa uma ressecção esofagiana por doença maligna são: estadiamento da neoplasia, condição clinica do paciente, via de acesso cirúrgico, extensão do esôfago a ser ressecado, extensão da linfadenectomia, tipo de reconstrução para substituição do esôfago e necessidade de ressecção gástrica associada nos tumores localizados no terço inferior.

Existem diversas vias disponíveis para serem utilizadas no acesso ao esôfago, cada uma delas com indicações específicas e que dependem basicamente da localização da neoplasia e da cirurgia proposta. Entre os principais tipos de abordagem descritas na literatura temos a técnica de Ivor Lewis (laparotomia e toracotomia direita com anastomose torácica), 
técnica de McKeown (Ivor Lewis modificada com cervicotomia e anastomose cervical), técnica de Sweet (incisão toracoabdominal esquerda com anastomose torácica), técnica transhiatal (laparotomia sem toracotomia) e ainda outros acessos minimamente invasivos, como a toracoscopia e a laparoscopia, que ainda se encontram em fase de desenvolvimento, necessitando de estudos prospectivos para melhor determinar respostas quanto à segurança, radicalidade e sobrevida, comparados com cirurgia convencional.

Atualmente, permanece a grande questão com relação à via de acesso cirúrgica mais adequada e à extensão do procedimento, já que não está definido se uma maior radicalidade cirúrgica pode alterar substancialmente a sobrevida e o intervalo livre de doença. As abordagens mais comumente utilizadas para ressecção do câncer do esôfago são: Transtorácica e Transhiatal. Os partidários da via transtorácica dão preferência a esta pela obtenção de melhor visualização para o estadiamento cirúrgico, maior facilidade para dissecção do órgão com menores riscos de lesões iatrogênicas e possibilidade de campo mais adequado para dissecção do grupamento linfonodal no mediastino superior e médio, mais difícil por via transhiatal. Dessa forma, um procedimento cirúrgico mais radical poderia promover remoção completa do tumor e seus linfonodos satélites, minimizando o potencial de doença residual e recidiva. Por outro lado, este tipo de acesso está associado com uma maior incidência de complicações pulmonares no pós-operatório, aumentando assim a morbidade do procedimento. Entretanto, os que dão preferência à via de acesso transhiatal sustentam que esta fornece uma radicalidade cirúrgica semelhante à via transtorácica, minimizando as complicações pulmonares pós-operatórias.

Quatro estudos randomizados importantes comparam os resultados da esofagectomia por via transtorácica e transhiatal ${ }^{19-22}$. Três destes estudos tiveram uma amostra muito pequena e assim, ficou impossibilitada a realização de qualquer conclusão definitiva. Em todos estes, as taxas de morbidade pós-operatória e de mortalidade foram semelhantes nas duas vias de acesso cirúrgico ${ }^{19-21}$. O quarto estudo envolveu 220 pacientes com adenocarcinoma do esôfago médio ou distal os quais foram submetidos aleatoriamente à esofagectomia por via transtorácica ou transhiatal. Observouse um maior número de linfonodos dissecados nos pacientes submetidos à esofagectomia por via transtorácica (31vs16, p<0.001), porém não houve diferença com relação ao número de cirurgias R0, R1 ou R2. Pode-se observar também uma maior incidência de complicações pulmonares e um maior tempo de internação hospitalar nos pacientes com via de acesso transtorácico, não se observando, porém, diferença significativa na mortalidade pós-operatória ${ }^{22}$. Assim, a escolha da via mais adequada depende de vários fatores, e entre eles, fatores técnicos, como por exemplo, a habilidade do cirurgião, e principalmente, as características individuais do paciente como localização, performance status e estadiamento da neoplasia. Fatores estes que foram utilizados no nosso estudo para escolha da via cirúrgica.

Outro aspecto a ser considerado é relacionado à extensão do procedimento cirúrgico, principalmente no tocante à linfadenectomia. Importante lembrar que a margem cirúrgica preconizada para o câncer de esôfago é de, no mínimo, $7 \mathrm{~cm}$ na margem proximal, e de $5 \mathrm{~cm}$ na margem distal. A extensão ideal da linfadenectomia continua sendo um tema de grande debate na maioria dos tumores sólidos, principalmente do trato gastrointestinal. No caso do câncer de esôfago, muitos cirurgiões não levam em conta o real valor da linfadenectomia, pois acreditam que o comprometimento linfonodal determina doença sistêmica e assim sem chance de cura para os pacientes. Infelizmente a nomeclatura para as estações linfonodais envolvendo o esôfago não é uniforme e existem diferentes estações linfonodais quando se compara a nomeclatura da American Joint Committe of Cancer (AJCC) e a nomeclatura da Sociedade Japonesa das Doenças Esofagianas. Esta diferença gera algumas dúvidas na definição da linfadenectomia padrão para o câncer de esôfago, dificultando a padronização do procedimento ${ }^{23,24}$.

Alguns estudos demonstraram que a ressecção em bloco envolvendo esôfago, pericárdio, ducto torácico, veia ázigos e pleura parietal bilateral (esofagectomia em bloco) levavam a uma redução na recidiva loco-regional com aumento da sobrevida a longo prazo dos pacientes portadores de câncer no esôfago ${ }^{25-27}$. Sua principal indicação, são para os pacientes com tumores do esôfago médio ou distal e que apresentem estadiamento I e II pelas normas do $\mathrm{TNM}^{9,10}$. Em casos selecionados pode ser realizado este tipo de ressecção em tumores com estadiamento mais avançado, tendo nestes casos resultados incertos e com benefício não comprovado na literatura.

Em algumas situações é realizada a dissecção dos linfonodos da cadeia cervical, mediastinal superior e celíaco (esofagectomia em três campos). Adeptos desta técnica reforçam que este procedimento aprimora o estadiamento cirúrgico (devido a uma maior amostragem linfonodal), e aumenta as taxas de cirurgia R0, contribuindo assim, para melhorar o prognóstico destes pacientes. O grande problema é que essa dissecção radical aumenta o tempo operatório e o risco de complicações pulmonares, sangramento, linfedema, lesão nervosa e fístulas ${ }^{15,16}$.

Análises mostrando o predomínio da recidiva sistêmica e mediastinal ao contrário da cervical, e a ausência de diferenças estatísticas entre a linfadenectomia em bloco e a linfadenectomia em três campos, torna a escolha do melhor procedimento uma questão ainda sem resposta na literatura.

A seleção do substituto esofágico apropriado depende da disponibilidade do órgão (ressecção ou cirurgias prévias), sua anatomia (suprimento vascular, tamanho comparado ao esofageano e comprimento) e sua aplicabilidade (expectativa de vida e condições gerais do paciente, dificuldade técnica da reconstrução, e as características funcionais do órgão a ser usado) ${ }^{28}$.

A preferência para reconstrução com o estômago decorre da sua grande vascularização, maior facilidade técnica e necessidade de somente uma anastomose, comparado com a necessidade de várias anastomoses, quando se reconstrói com o cólon. A desvantagem do tubo gástrico inclui o risco de isquemia da parte mais distal do fundo gástrico, podendo levar a necrose do tubo, deiscência ou posteriormente, estenose da anastomose esôfago-gástrica, e ainda a exposi- 
ção do epitélio escamoso do esôfago remanescente a secreção ácida secretada pelo estômago, podendo assim levar ao Esôfago de Barrett e ao adenocarcinoma ${ }^{29}$.

Em caso de impossibilidade de se confeccionar o tubo gástrico, a interposição colônica pode ser uma opção apropriada, dando-se sempre preferência ao cólon transverso devido à sua rica vascularização e mais fácil mobilização. As principais vantagens da reconstrução com o tubo colônico, decorrem da resistência da mucosa colônica ao refluxo ácido do estômago, minimizando o risco do Esôfago de Barrett e ao adenocarcinoma; além de uma menor incidência de estenose da anastomose esôfago-colônica, quando comparado com a anastomose esôfago-gástrica ${ }^{28}$.

Outro aspecto de grande importância corresponde à via pela qual o tubo confeccionado após esofagectomia irá ascender para chegar à região cervical. O acesso pelo mediastino posterior tem a preferência de vários autores, apresentando como vantagem a maior facilidade técnica, por ser mais anatômico e de trajeto mais curto. Outros acessos que podem ser utilizados são o retroesternal e o subcutâneo, sendo indicado principalmente na impossibilidade da via mediastinal ${ }^{30}$.

Neste estudo, a mortalidade operatória foi baixa e a morbidade operatória foi elevada, principalmente com relação às complicações respiratórias, comprovando os dados levantados na literatura especializada em cirurgias no esôfago. Assim podemos concluir que a esofagectomia transtorácica ou transhiatal e a reconstrução com o tubo gástrico com anastomose cervical é factível tecnicamente, sendo um procedimento realizado de rotina nos pacientes portadores de câncer do esôfago com indicação cirúrgica matriculados na Seção de Cirurgia Abdômino-pélvica do INCA. Contudo, convém ressaltar que a reconstrução com tubo colônico esta indicada principalmente para os pacientes sem possibilidade de utilização do estômago para reconstrução do trato alimentar, sendo uma opção válida e com excelentes resultados.

\begin{abstract}
Background: To describe the main reconstruction techniques of the alimentary tract after esophagectomy due to esophagus cancer in patients enrolled. Methods: We analyzed retrospectively 68 patients submitted to the esophagectomy between february of 1997 and november of 2005. All of the patients included in the study were submitted to the reconstruction with gastric tube and colon segment and cervical anastomosis. Results: The mean age was 55,4 years (25-74 years). 50 patients were of male gender and 18 patients of female gender. 27 patients presented the tumor localized in the medium esophagus and 41 patients in the distal esophagus, being epidermoid carcinoma in 35 patients and adenocarcinoma in 33 patients. The ressection was performed through transthoracic access in 35 individuals and through transhiatal access in 33. The reconstruction with gastric tube was performed in 58 patients and with colonic tube in 10 patients. The series total morbidity was 52,9\%. The operative mortality was 5,8\%. The mean survival was of 35 months. Conclusion: The esophagectomy with reconstruction with gastric tube and cervical anastomosis is feasible technically; being a routine procedure realized in the patients carriers of esophagus cancer with surgical indication. The reconstruction with the colonic tube is indicated mainly for the patients without possibility of the stomach use for reconstruction of the alimentary tract, being a valid option and with excellent results.
\end{abstract}

Key words: Esophageal neoplasms; Gastrointestinal Tract; Esophagectomy; Gastroplasty.

\section{REFERÊNCIAS}

1. Patel AN, Buenaventura PO. Current staging of esophageal carcinoma. Surg Clin North Am. 2005; (85):555-67.

2. Enzinger PC, Mayer RJ. Esophageal cancer. N Engl J Med. 2003; 349(23):2241-52.

3. Eloubeidi MA, Mason AC, Desmond RA, El-Serag HB. Temporal trends (1973-1997) in survival of patients with esophageal adenocarcinoma in the United States: a glimmer of hope? Am J Gastroenterol. 2003; 98(7):1627-33.

4. McLarty AJ, Deschamps C, Trastek VF, Allen MS, Pairolero PC, Harmsen WS. Esophageal resection for cancer of the esophagus: long-term function and quality of life. Ann Thorac Surg. 1997; 63(6): 1568-72.

5. Patel AN, Preskitt JT, Kuhn JA, Hebeler RF, Wood RE, Urschel HC Jr. Surgical management of esophageal carcinoma. Proc (Bayl Univ Med Cent). 2003; 16(3): 280-4.
6. Wu PC, Posner MC. The role of surgery in the management of oesophageal cancer. Lancet Oncol. 2003; 4(8):481-8.

7. Ando N, Ozawa S, Kitagawa Y, Shinozawa Y, Kitajima M. Improvement in the results of surgical treatment of advanced squamous esophageal carcinoma during 15 consecutive years. Ann Surg. 2000; 232(2):225-32.

8. Hagen JA, DeMeester SR, Peters JH, Chandrasoma P, DeMeester TR. Curative resection for esophageal adenocarcinoma: analysis of 100 en bloc esophagectomies. Ann Surg. 2001; 234(4):520 30; discussion 530-1.

9. Altorki N, Skinner D. Should en bloc esophagectomy be the standard of care for esophageal carcinoma? Ann Surg. 2001; 234(5):581-7.

10- Skinner DB, Little AG, Ferguson MK, Soriano A, Staszak VM. Selection of operation for esophageal cancer based on staging. Ann Surg. 1986; 204(4):391-401. 
11- Altorki NK, Girardi L, Skinner DB. En bloc esophagectomy improves survival for stage III esophageal cancer. J Thorac Cardiovasc Surg. 1997; 114(6):948-55; discussion 955-6.

12- Collard JM, Otte JB, Fiasse R, Laterre PF, De Kock M, Longueville J, Glineur D, Romagnoli R, Reynaert M, Kestens PJ. Skeletonizing en bloc esophagectomy for cancer. Ann Surg. 2001; 234(1):25-32.

13- Devesa SS, Blot WJ, Fraumeni JF Jr. Changing patterns in the incidence of esophageal and gastric carcinoma in the United States. Cancer. 1998; 83(10):2049-53.

14- Jemal A, Murray T, Ward E, Samuels A, Tiwari RC, Ghafoor A, Feuer EJ, Thun MJ. Cancer statistics, 2005. CA Cancer J Clin. 2005; 55(1):10-30.

15- Akiyama $H$, Tsurumaru M, Udagawa H, Kajiyama Y. Radical lymph node dissection for cancer of the thoracic esophagus. Ann Surg. 1994; 220(3):364-72; discussion 372-73.

16- Altorki N, Kent M, Ferrara C, Port J. Three-field lymph node dissection for squamous cell and adenocarcinoma of the esophagus. Ann Surg. 2002; 236(2): 1770-83.

17- Kodama M, Kakegawa T. Treatment of superficial cancer of the esophagus: a summary of responses to a questionnaire on superficial cancer of the esophagus in Japan. Surgery. 1998; 123(4):4329.

18- Law S, Wong J. Lymph node dissection in surgical treatment of esophageal neoplasms. Surg Oncol Clin North Am. 2007; 16(1):115-31.

19- Goldminc M, Maddern G, Le Prise E, Meunier B, Campion JP, Launois B. Oesophagectomy by transhiatal approach or thoracotomy: a prospective randomized trial. Br J Surg. 1993; 80(3):367-70.

20- Chu KM, Law SY, Fok M, Wong J. A prospective randomized comparison of transhiatal and transthoracic resection for lowerthird esophageal carcinoma. Am J Surg. 1997; 174(3):320-4.

21- Jacobi CA, Zieren HU, Müller JM, Pichlmaier H. Surgical therapy of esophageal carcinoma: the influence of surgical approach and esophageal resection on cardiopulmonary function. Eur $\mathbf{J}$ Cardiothorac Surg. 1997; 11(1):32-7.

22- Hulscher JB, van Sandick JW, de Boer AG, Wijnhoven BP, Tijssen JG, Fockens P, Stalmeier PF, ten Kate FJ, van Dekken H, Obertop H, Tilanus HW, van Lanschot JJ. Extended transthoracic resection compared with limited transhiatal resection for adenocarcinoma of the esophagus. N Engl J Med. 2002; 347(21):1662-9.
23- American Joint Committee on Cancer. Esophagus. In: Greene FL, editor. AJCC cancer staging manual/American Joint Committee on Cancer. New York: Springer; 2002. p. 91-5.

24- Japanese Society for Esophageal Diseases. Guidelines for clinical and pathologic studies on carcinoma of the esophagus. $9^{\text {th }}$ edition. Tokyo: Kanehara \& Co.; 2001.

25- Tachibana M, Kinugasa S, Yoshimura H, Shibakita M, Tonomoto Y, Dhar DK, Tabara H, Nagasue N. En-bloc esophagectomy for esophageal cancer. Am J Surg. 2004; 188(3):254-60.

26- Clark GW, Peters JH, Ireland AP, Ehsan A, Hagen JA, Kiyabu MT, Bremner CG, DeMeester TR. Nodal metastasis and sites of recurrence after en bloc esophagectomy for adenocarcinoma. Ann Thorac Surg. 1994; 58(3):646-53; discussion 653-4.

27- Oberg S, Johansson J, Wenner J, Walther B. Metaplastic columnar mucosa in the cervical esophagus after esophagectomy. Ann Surg. 2002; 235(3):338-45.

28- Ferguson MK. Reconstructive surgery of the esophagus. In: Ferguson MK. Routes for esophageal replacement. Armonk (NY): Futura Publishing; 2002. p.117-27.

29- Briel JW, Tamhankar AP, Hagen JA, DeMeester SR, Johansson J, Choustoulakis E, Peters JH, Bremner CG, DeMeester TR Prevalence and risk factors for ischemia, leak, and stricture of esophageal anastomosis: gastric pull-up versus colon interposition. J Am Coll Surg. 2004; 198(4):536-41; discussion 541-2.

30- Pinto CE, Pinheiro RN, Kesley R. Morbidity and mortality after esophagectomy of cancer in Brazilian National Cancer Institute [abstract]. Dis Esophagus. 2004; 17 Suppl 1:A149.

Como citar este artigo:

Pinto CE, Sá EA, Fernandes DS, Dias JA. Experiência do Inca na reconstrução do trato alimentar com tubo gástrico ou colônico na esofagectomia por câncer de esôfago. Rev Col Bras Cir. [periódico na Internet] 2008; 35(6). Disponível em URL: http://www.scielo.br/ rcbc

Endereço para correspondência:

Carlos Eduardo Pinto

Av. Canal de Marapendi, 2915 / 105 B.3

Barra da Tijuca

22631-05- Rio de Janeiro - RJ

E-mail: caudu2003@yahoo.com.br 